\title{
Ultrasmall TPGS-PLGA Hybrid Nanoparticles for Site-Specific Delivery of Antibiotics into Pseudomonas aeruginosa Biofilms in Lungs
}

Wan, Feng; Bohr, Søren S.-R.; Kodziska, Sylvia Natalie; Jumaa, Haidar; Huang, Zheng; Nylander, Tommy; Thygesen, Mikkel Boas; Sørensen, Kasper Kildegaard; Jensen, Knud Jørgen; Sternberg, Claus Total number of authors:

12

Published in:

ACS Applied Materials and Interfaces

Link to article, DOI:

10.1021/acsami.9b19644

Publication date:

2020

Document Version

Peer reviewed version

Link back to DTU Orbit

Citation (APA):

Wan, F., Bohr, S. S-R., Kodziska, S. N., Jumaa, H., Huang, Z., Nylander, T., Thygesen, M. B., Sørensen, K. K., Jensen, K. J., Sternberg, C., Hatzakis, N., \& Mørck Nielsen, H. (2020). Ultrasmall TPGS-PLGA Hybrid Nanoparticles for Site-Specific Delivery of Antibiotics into Pseudomonas aeruginosa Biofilms in Lungs. ACS Applied Materials and Interfaces, 12(1), 380-389. https://doi.org/10.1021/acsami.9b19644

\section{General rights}

Copyright and moral rights for the publications made accessible in the public portal are retained by the authors and/or other copyright owners and it is a condition of accessing publications that users recognise and abide by the legal requirements associated with these rights.

- Users may download and print one copy of any publication from the public portal for the purpose of private study or research.

- You may not further distribute the material or use it for any profit-making activity or commercial gain

- You may freely distribute the URL identifying the publication in the public portal 


\section{ACS APPLIED MATERIALS \\ INTERFACES}

\section{Biological and Medical Applications of Materials and Interfaces}

\section{Ultra-small TPGS-PLGA Hybrid Nanoparticles for Site-specific Delivery of Antibiotics into Pseudomonas Aeruginosa Biofilms in Lungs}

Feng Wan, Søren S.-R. Bohr, Sylvia Natalie K\#odzi\#ska, Haidar Jumaa, Zheng

Huang, Tommy Nylander, Mikkel B. Thygesen, Kasper Kildegaard Sørensen,

Knud J. Jensen, Claus Sternberg, Nikos S. Hatzakis, and Hanne Mørck Nielsen

ACS Appl. Mater. Interfaces, Just Accepted Manuscript • DOI: 10.1021/acsami.9b19644 • Publication Date (Web): 05 Dec 2019

Downloaded from pubs.acs.org on December 6, 2019

\section{Just Accepted}

"Just Accepted" manuscripts have been peer-reviewed and accepted for publication. They are posted online prior to technical editing, formatting for publication and author proofing. The American Chemical Society provides "Just Accepted" as a service to the research community to expedite the dissemination of scientific material as soon as possible after acceptance. "Just Accepted" manuscripts appear in full in PDF format accompanied by an HTML abstract. "Just Accepted" manuscripts have been fully peer reviewed, but should not be considered the official version of record. They are citable by the Digital Object Identifier (DOI®). "Just Accepted" is an optional service offered to authors. Therefore, the "Just Accepted" Web site may not include all articles that will be published in the journal. After a manuscript is technically edited and formatted, it will be removed from the "Just Accepted" Web site and published as an ASAP article. Note that technical editing may introduce minor changes to the manuscript text and/or graphics which could affect content, and all legal disclaimers and ethical guidelines that apply to the journal pertain. ACS cannot be held responsible for errors or consequences arising from the use of information contained in these "Just Accepted" manuscripts. 


\title{
Ultra-small TPGS-PLGA Hybrid Nanoparticles for Site-specific Delivery of Antibiotics into Pseudomonas Aeruginosa Biofilms in Lungs
}

\author{
Feng Wan 1; Søren Schmidt-Rasmussen Bohr ${ }^{2,}$; Sylvia Natalie Kłodzińska ${ }^{1}$; Haidar Jumaa 1; Zheng \\ Huang 1; Tommy Nylander 4; Mikkel Boas Thygesen 2, 3; Kasper Kildegaard Sørensen 2, 3; Knud Jørgen \\ Jensen 2, 3; Claus Sternberg 5; Nikos Hatzakis 2,3 *; Hanne Mørck Nielsen 1,6 * \\ ${ }^{1}$ Department of Pharmacy, University of Copenhagen, Universitetsparken 2, DK-2100 Copenhagen Ø, \\ Denmark; ${ }^{2}$ Department of Chemistry \& Nano-Science Center, University of Copenhagen, \\ Thorvaldsensvej 40, DK-1871 Frederiksberg C, Denmark; ${ }^{3}$ Novo Nordisk Foundation Centre for \\ Protein Research, Faculty of Health and Medical Sciences, University of Copenhagen, Blegdamsvej \\ 3B, 2200 Copenhagen, Denmark; ${ }^{4}$ Department of Physical Chemistry, Lund University, SE-221 00 \\ Lund, Sweden; ${ }^{5}$ Department of Biotechnology and Biomedicine, Technical University of Denmark, \\ Building 221, Søltofts Plads, DK-2800 Lyngby, Denmark; ${ }^{6}$ Center for Biopharmaceuticals and \\ Biobarriers in Drug Delivery, Department of Pharmacy, University of Copenhagen, Universitetsparken \\ 2, DK-2100 Copenhagen Ø, Denmark
}

*Corresponding author: hanne.morck@sund.ku.dk; hatzakis@,nano.ku.dk 


\begin{abstract}
Inhaled antibiotic treatment of cystic fibrosis $(\mathrm{CF})$-related bacterial biofilm infections is challenging due to the pathological environment of the lungs. Here, we present an 'environment adaptive' nanoparticle composed of a solid poly lactic-co-glycolic acid (PLGA) core and a mucus-inert, enzymatically cleavable shell of $d$ - $\alpha$-tocopheryl polyethylene glycol 1000 succinate (TPGS) for sitespecific delivery of antibiotics to bacterial biofilms via aerosol administration. The hybrid nanoparticles with ultra-small size were self-assembled via a nanoprecipitation process by using a facile microfluidic method. The interactions of the nanoparticles with the biological barriers were comprehensively investigated by using cutting-edge techniques (e.g. quartz crystal microbalance with dissipation monitoring, total internal reflection fluorescence microscopy-based particle tracking, in vitro biofilm model cultured in a flow-chamber system, and quantitative imaging analysis). Our results suggest that the mucus-inert, enzymatically cleavable TPGS shell enables the nanoparticles to penetrate through the mucus, accumulate in the deeper layer of the biofilms, and serve as sustained release depot, thereby, improving the killing efficacy of azithromycin (a macrolide antibiotic) against biofilm-forming Pseudomonas aeruginosa. In conclusion, the ultra-small TPGS-PLGA hybrid nanoparticles represent an efficient delivery system to overcome the multiple barriers and release antibiotics with a sustained manner in the vicinity of the biofilm-forming bacteria.
\end{abstract}

Keywords: TPGS-PLGA hybrid nanoparticles, aerosol administration, cystic fibrosis, Pseudomonas aeruginosa biofilm, Bio-Nano interaction 


\section{Introduction}

Chronic Pseudomonas aeruginosa (P. aeruginosa) infection is characteristic of cystic fibrosis $(\mathrm{CF})$, which usually leads to accelerated morbidity and mortality of CF patients ${ }^{1-2}$. Antibiotic treatment is a cornerstone of CF therapy to reduce bacterial load, exacerbation rates and loss of pulmonary function. However, the formation of persistent bacterial biofilm challenges the conventional antibiotic treatments that rely on parenteral and oral administration, because the minimal inhibitory concentration (MIC) and the minimal bactericidal concentration $(\mathrm{MBC})$ of an antibiotic to biofilm-growing bacteria can be 1001000 fold higher than to planktonic bacteria ${ }^{3}$. Aerosol administration of antibiotics potentially provides high drug concentration at the site of infection and minimizes systemic side effects ${ }^{4-6}$. However, the abnormally thick and viscous mucus layer in CF patients hinders the diffusion of inhaled antibiotics into the regions with high bacterial loads ${ }^{7-8}$, consequently, resulting in limited efficacy. In addition, extracellular polymeric substances (EPS) produced by bacteria and composed of exopolysaccharides, proteins, enzymes, lipids and nucleic acids, present a cohesive, three-dimensional polymer network that can impede the penetration of drugs into biofilm ${ }^{9}$. Furthermore, the EPS matrix may also act as an external degradative system, enabling the deactivation of antibiotics through binding interactions in these environments ${ }^{10}$.

Nanoparticle-based delivery strategies are being explored to promote the delivery of antibiotics into bacterial biofilms ${ }^{11-12}$. In general, nanoparticle size plays an important role in overcoming the steric hindrance of mucus and EPS ${ }^{13-14}$. As reported, the 3D mesh spacing of fresh, undiluted and unaltered CF sputum is in the range of $60-300 \mathrm{~nm}{ }^{15}$. Nanoparticles with size less than $100 \mathrm{~nm}$ seem to be optimal for biofilm-penetrating ${ }^{14}$. In addition, variety of surface engineered nanoparticles has been developed to reduce the adhesive interaction of nanoparticles with mucus. In recent works, $\mathrm{pH}-$ 
sensitive polymeric nanoparticles with surface charge-switching property (i.e. presenting negativelycharged surfaces at physiological condition, however, switching the surface to positive charge when exposed to bacterial infection-related acidic $\mathrm{pH}$ levels) demonstrated great potential for targeting delivery of antibiotics to sites of infections via systemic administration ${ }^{16-18}$. In spite of the great promising demonstrated at the tissue level (i.e., in vitro cultured bacterial biofilms), the surface chargeadaptive approach based on $\mathrm{pH}$-sensitive polymers may be not applicable for aerosol antibiotics delivery due to the complicated microenvironment in lungs of $\mathrm{CF}$ patients, such as the acidic $\mathrm{pH}$ level (approximately 5.5-6.5) and the elevated salt concentration of lung lining fluid ${ }^{19-22}$. Because the pHsensitive polymeric nanoparticles could switch their charge prior to reaching to the bacterial biofilms, consequently, leading to the mistargeting of the nanoparticles. So far, the effective approach for sitespecific delivery of antibiotics into CF-related biofilms via aerosol administration is highly unmet and rarely reported.

Here, we present an environment adaptive nanoparticles delivery system by exploiting the amphiphilic structure and enzymatic cleavage of d- $\alpha$-tocopheryl polyethylene glycol 1000 succinate (TPGS). TPGS is an FDA-approved pharmaceutical excipient consisting of a lipophilic moiety (vitamin E) and a hydrophilic moiety (PEG chain) ${ }^{23-24}$, which is documented to be cleaved into vitamin E and PEG by enzymes (e.g. esterase) ${ }^{25}$. It is known that both clinical and environmental $P$. aeruginosa strains are able to produce large array of extracellular enzymes, including esterase ${ }^{26-27}$. Therefore, we expect that nanoparticles with a TPGS shell (with the PEG chain exposed towards the aqueous bulk phase) could allow the nanoparticles for penetrating through the biological barriers (e.g. mucus, EPS) into bacterial biofilms due to the low nonspecific binding of PEG ${ }^{13,28}$. Subsequently, the enzymatic cleavage of TPGS in the biofilms could generate a lipophilic surface (vitamin E) of the nanoparticles, which 
enables the nanoparticles to anchor in the biofilm and serve as antibiotic depots, thus prolonging the exposure time of bacteria to antibiotics. To prove the concept, we fabricated TPGS-poly (lactide-coglycolide) (PLGA) hybrid nanoparticles via the microvortex-induced nanoprecipitation process in a microfluidic chip, where the assembly of TPGS in the aqueous phase with PLGA in the organic phase results in a PLGA core surrounded by a TPGS shell with the PEG chain exposed towards the aqueous bulk phase. In addition, we comprehensively investigated the interactions of TPGS-PLGA hybrid nanoparticles with the biological barriers (e.g. mucus, bacterial biofilm, and bacterial membrane) using a variety of cutting-edge techniques (e.g. quartz crystal microbalance with dissipation monitoring (QCM-D), total internal reflection fluorescence microscopy (TIRFM)-based particle tracking, in vitro biofilm model cultured in a flow-chamber system, and quantitative imaging analysis). Ultimately, azithromycin (AZI, a macrolide antibiotic), which has previously demonstrated pleiotropic effects on P. aeruginosa ${ }^{29}$, was used to demonstrate the nanoparticle-mediated anti-biofilm efficacy.

\section{Experimental Section}

Materials: PLGA (Resomer ${ }^{\circledR}$ RG503H, L:G ratio of 50:50, molecular weight 24,000-38,000 Da), D$\alpha$-tocopheryl polyethylene glycol 1000 succinate (TPGS), mercaptoundecanoic acid (MUA), Nhydroxysuccinimide (NHS), $N$-(3-dimethylaminopropyl)- $N$ '-ethylcarbodiimide hydrochloride (EDC), ethanolamine hydrochloride (ETA-HCl), 11-porcine stomach mucin (Type III, bound sialic acid 0.51.5\%, partially purified powder), Mueller Hinton Broth (MHB), LB Broth, deoxyribonucleic acid (DNA) from fish sperm, glucose, crystal violet, ethannol (HPLC grade), acetonitrile (HPLC grade), Sodium phosphate monobasic $\left(\mathrm{NaH}_{2} \mathrm{PO}_{4}\right)$, Sodium chloride $(\mathrm{NaCl})$, citric acid, 4-(2-hydroxyethyl)-1- 
piperazineethanesulfonic acid (HEPES), and tris(hydroxymethyl)aminomethane (Tris) were purchased from Sigma-Aldrich (Broendby, Denmark). 1,1'-dioctadecyl-3,3,3',3'-tetramethylindocarbocyanine perchlorate $\left(\mathrm{DiIC}_{18(3)}\right)$, and propidium iodide (PI) were purchased from Invitrogen (Carlsbad, CA, USA) and Molecular Probes (Eugene, OR, USA), respectively. 1-Palmitoyl-2-oleoyl-sn-glycero-3phosphoethanolamine (POPE) and 1-palmitoyl-2-oleoyl-sn-glycero-3-phospho-(1'-rac-glycerol) (POPG) were purchased from Avanti Polar Lipids (Alabaster, AL, USA). Azithromycin dihydrate (AZI, 97\%) was bought from Fluorochem (Hadfield, UK). P. aeruginosa (PA O1) and P. aeruginosa (PAO1 tagged with GFP) were kindly provided by the Institute of Immunology and Microbiology (University of Copenhagen) and the Department of Biotechnology and Biomedicine (Technical University of Denmark), respectively. Ultrapure water was obtained from a PURELAB ${ }^{\circledR}$ flex machine (ELGA LabWater, High Wycombe, UK).

Preparation and characterization of nanoparticles: TPGS-PLGA hybrid nanoparticles were prepared by using a microfluidic method as previously reported ${ }^{30}$. Briefly, the organic phase, composed of $1 \%(\mathrm{w} / \mathrm{v})$ PLGA in acetonitrile, and the aqueous phase, composed of TPGS $(0.01 \% \sim 0.2 \%, w / v)$ in HEPES buffer $(10 \mathrm{mM}, \mathrm{pH} 7.4)$ (without TPGS for preparation of PLGA nanoparticles) were pumped by using syringes (Gastight 1010, Hamilton Robotics, Reno, NV, USA) into the inner channel (flow rate $1 \mathrm{~mL} / \mathrm{min}$ ) and outer channels $(5 \mathrm{~mL} / \mathrm{min}$ ) of the microfluidic chip, respectively. The resulting nanoparticle dispersion was collected in a beaker and stored in a dark room under magnetic stirring for $4 \mathrm{~h}$ to evaporate the organic solvent.

For the preparation of the fluorescent nanoparticles for the investigation of mucus-penetrating property using particle tracking, Atto655-conjugated PLGA was synthesized (supporting information (SI), Figure S1 and Figure S2) and used to label the nanoparticles by addition of Atto655-conjugated PLGA 


\begin{abstract}
$(2 \% \mathrm{w} / \mathrm{w}$ to PLGA) to the organic phase. For the preparation of the fluorescent nanoparticles for the investigation of the nanoparticle penetration into the bacterial biofilms, DiIC $_{18(3)}$ was added to the organic phase to a final concentration of $0.005 \%(\mathrm{w} / \mathrm{v})$. For the preparation of antimicrobial nanoparticles (AZI-loaded TPGS-PLGA hybrid nanoparticles), AZI was dissolved in the organic phase to the concentration of $0.2 \%(\mathrm{w} / \mathrm{v})$. The unencapsulated AZI was then removed by centrifugation (4000×g) using an Amicon ${ }^{\circledR}$ filter (MWCO 100 kDa, Merck, Darmstadt, Germany).
\end{abstract}

Size and zeta potential of the nanoparticles were measured by using dynamic light scattering and laser Doppler electrophoresis, respectively. The samples were diluted using HEPES buffer (10 mM, pH 7.4) to a concentration of $0.1 \mathrm{mg} / \mathrm{mL}$ prior to measurements, which were carried out at $25{ }^{\circ} \mathrm{C}$ by using a Zetasizer Nano ZS (Malvern Instruments, Worcestershire, UK) equipped with a $633 \mathrm{~nm}$ laser and $173^{\circ}$ detection optics. Data acquisition and analysis were performed by using the Malvern DTS v6.30 software (Malvern Instruments). The morphological analysis of the nanoparticles was carried out by using a Tecnai G2 20 TWIN transmission electron microscope (FEI, Hillsboro, OR, USA). The nanoparticle sample in ultrapure water $(100 \mu \mathrm{L})$ was mounted onto a TEM grid, followed by staining with $1 \%(\mathrm{w} / \mathrm{v})$ ammonium molybdate for $1 \mathrm{~min}$. The grid was dried by removing the liquid using a filter paper. All of the observations were made in bright field mode at an acceleration voltage of $10 \mathrm{kV}$. The digital images were recorded with a Gatan Imaging Filter 100 CCD camera (Gatan, Pleasanton, CA, USA).

Encapsulation efficiency, drug loading, and drug release: To determine the drug loading and the encapsulation efficiency of AZI in the nanoparticles, the purified AZI-loaded TPGS-PLGA hybrid nanoparticles in suspension $(50 \mu \mathrm{L})$ were dissolved in acetonitrile $(250 \mu \mathrm{L})$. The amount of AZI, TPGS and PLGA were analyzed by using high performance liquid chromatography with evaporative light 
scattering detector (ELSD-HPLC) (SI, Table S1, and Figure S3). The average encapsulation efficiency $(E E \%)$ and the drug loading $(D L \%)$ were calculated by using the eq. 1 and 2, respectively:

$E E \%=\frac{m_{A Z I}}{M_{A Z I}} \times 100 \%$

$D L \%=\frac{m_{A Z I}}{m_{A Z I}+m_{P L G A}} \times 100 \%$

Where $E E \%$ is the encapsulation efficiency; $D L \%$ is the drug loading of AZI into nanoparticles; $M_{\mathrm{AZI}}$ is the amount of AZI in the non-purified nanoparticles dispersion, $m_{\mathrm{AZI}}$ is the content (mass) of AZI in the purified nanoparticles; $m_{\mathrm{PLGA}}$ is the content (mass) of PLGA in the purified nanoparticles.

In vitro drug release of AZI from the nanoparticles was investigated by using a dialysis-bag method. Briefly, $1 \mathrm{~mL}$ of AZI-loaded TPGS-PLGA hybrid nanoparticles dispersion (with a total drug concentration of $500 \mu \mathrm{g} / \mathrm{mL}$ ) was transferred into dialysis cassettes (MWCO $100 \mathrm{kDa}$, Thermo Fisher Scientific, Waltham, MA, USA). As a control, $1 \mathrm{~mL}$ of AZI solution (500 $\mu \mathrm{g} / \mathrm{mL})$ in HEPES buffer (pH 7.4) was injected into a separate dialysis cassette. The cassettes were then immersed into a $4 \mathrm{~mL}$ reservoir of HEPES buffer solution $(\mathrm{pH} 7.4)$ in an incubator at $37{ }^{\circ} \mathrm{C}$, with shaking using a linear shaking bath system (GLS400, Grant Instruments, Germany). The release medium was replaced with 4 $\mathrm{mL}$ fresh HEPES buffer solution $(\mathrm{pH} \mathrm{7.4)}$ at pre-determined time intervals. The amount of drug in the collected release medium was assayed by using an HPLC method.

Total internal reflection fluorescence microscopy (TIRFM)-based particle tracking: Particle tracking experiments were performed using a total internal reflection fluorescence microscope (IX 83, Olympus, Tokyo, Japan) using a EMCCD camera (ImagEM X2, Hamatsu, Hamamatsu City, Japan) and an oil immersion 100× objective (UAPON 100XOTIRF, Olympus, Tokyo, Japan), resulting in a 
resolution of $160 \mathrm{~nm}$ per pixel. Atto655-labeled PLGA nanoparticles were excited using a $655 \mathrm{~nm}$ solid state laser line (Cellsens, Olympus, Tokyo, Japan) with an exposure time of $50 \mathrm{~ms}, 200 \mathrm{~nm}$ penetration depth and 300 EM gain. A 700/75 nm ET band pass filter was used to filter emission signal. All experiments were performed at $37^{\circ} \mathrm{C}$. The TIRFM setup allows for real-time monitoring of individual particle characteristics and behaviors in close proximity to the surface (i.e., evanescent plane) by eliminating signals from the bulk sample.

Penetration experiments. To measure the rate of nanoparticle penetration, $20 \mu \mathrm{L}$ biosimilar mucus prepared as described previously ${ }^{31}$ (pH 6.5) was added to a custom-built Teflon ${ }^{\mathrm{TM}}$ chamber designed to support $Ø 25$ microscopy glass slides, resulting in a mucus layer of approximately $2 \mathrm{~mm}$. The nanoparticles were mixed with mucus in a 1:10 ratio (v:v) to, a) dilute the nanoparticle dispersion and to b) avoid further dilution of the mucus within the chamber upon addition of the sample. The freshly prepared mucus-nanoparticle dispersion was vortexed and allowed to equilibrate $10 \mathrm{~min}$ at $37{ }^{\circ} \mathrm{C}$ prior to imaging. Subsequently, the image acquisition was initiated once the mucus-nanoparticle dispersion $(15 \mu \mathrm{L})$ was carefully added on top of the mucus layer in the chamber. The imaging at the deposition surface was recorded at a frame rate of $1 \mathrm{~s}^{-1}$, enabling long term experiments and thus extraction of arrival times through the mucus.

Tracking experiments. To further investigate the interaction of nanoparticles with mucus and bacterial membrane, we set up an assay to track the movement of single particles at the interface between mucus and bacterial membrane. Prior to imaging, a mixture of nanoparticle and mucus was made as described above and after equilibration added to a microscopy chamber (total dispersion volume of $20 \mu \mathrm{L}$ ). Image acquisition was performed to record the ultra-fast behavior at a frame rate of $15.2 \mathrm{~s}^{-1}$. To investigate the nanoparticles interaction with bacterial membrane, the glass slide of the microscopy 
chamber was coated with lipid bilayer composed of POPE and POPG (3:1, molar ratio) by using the previously reported approach ${ }^{32}$, which is usually used for mimicking outer membrane of $P$. aeruginos $a^{33}$. The diffusion parameters of the nanoparticles were obtained by analyzing the moving trajectory of the nanoparticles at the interface between mucus and bacterial membrane.

Image analysis. Quantitative image analysis was performed using a combination of the ImageJ plugin TrackMate (for localization of particles) and custom routines developed in-house using $\mathrm{R}$ and Python 34. Particles were located in the xy-plane using a Laplacian of Gaussian (LoG) approach ${ }^{35-36}$, which allows sub-pixel localization and tracking. For both penetration experiment and diffusion experiment, only particles residing in the observable zone for 10 frames or more would be used for analysis. To avoid the introduction of falsely connected particles, a maximum distance (step size) between consecutive frames of $0.96 \mu \mathrm{m}$ was applied. The setup of the penetration experiment, due to the low framerate, allows for the long term measurements $(>40 \mathrm{~min})$ of particles arriving at the evanescent field (observable zone), thus, directly providing a correlation to time of penetration. However, during the penetration experiments, particles cannot be tracked individually and thus the number of arrived particles was counted instead. In addition, to further decipher the interaction of the nanoparticles with the mucus, a spatial analysis of particle density is conducted by using the Ripley's K function (SI, Figure S4) ${ }^{37}$. For the tracking experiments, the mean square displacement (MSD) of each individual particle was calculated as earlier shown ${ }^{38-39}$ :

$\operatorname{MSD}(\tau=\mathrm{n} * \Delta \mathrm{t})=\left\langle\mathrm{r}^{2}(\tau)>* \frac{1}{\mathrm{~N}-\mathrm{n}} * \sum_{\mathrm{i}=1}^{\mathrm{N}-\mathrm{n}}\left(\mathrm{x}_{\mathrm{i}+\mathrm{n}}-\mathrm{x}_{\mathrm{i}}\right)^{2}+\left(\left(\mathrm{y}_{\mathrm{i}+\mathrm{n}}-\mathrm{y}_{\mathrm{i}}\right)\right)^{2}\right.$ 
Where $\Delta t$ is the time interval, $\mathrm{N}$ the number of total frames and $\mathrm{x}_{\mathrm{i}}$ and $\mathrm{y}_{\mathrm{i}}$ are coordinates at $\mathrm{t}=\mathrm{i}$. From the MSD, the instantaneous diffusion coefficient was calculated by fitting a straight line to the initial lag times ${ }^{40}$.

Quartz crystal microbalance with dissipation monitoring (QCM-D): QCM-D measurements were performed with an E4 system from Q-Sense (Gothenburg, Sweden) using carboxylic acidfunctionalized, gold-coated quartz crystals with a fundamental frequency of $4.95 \mathrm{~Hz}$ (QSX301, QSense). To deposit a stable mucin layer on the crystals, mucin was immobilized on the crystals by using an amine-coupling method. Briefly, the crystals were first functionalized with a monolayer of carboxylic-acid by immersion in a $1 \mathrm{mM}$ ethanolic solution of MUA for at least $12 \mathrm{~h}$ at room temperature. The resulting functionalized gold-coated quartz crystals were subsequently rinsed with ethanol, dried and mounted in the QCM-D chamber. While mounted in the chamber, the crystals were exposed to an aqueous solution of $200 \mathrm{mM}$ EDC and $50 \mathrm{mM}$ NHS for $10 \mathrm{~min}$, followed by rinsing with ultrapure water and citrate buffer (10 mM, pH 4.0). Afterwards, $0.5 \mathrm{mg} / \mathrm{mL}$ mucin in citrate buffer was applied for $30 \mathrm{~min}$, followed by rinsing with the citrate buffer for $10 \mathrm{~min}$. Unreacted NHS-ester was deactivated by rinsing with $1 \mathrm{M}$ ETA- $\mathrm{HCl}$ (in Tris buffer, $10 \mathrm{mM}, \mathrm{pH} 8.5$ ) for $15 \mathrm{~min}$. Following another rinsing with phosphate buffer (PBS, $\mathrm{pH} 6.25$, ionic strength $150 \mathrm{mM}$ ) for $10 \mathrm{~min}$, nanoparticle dispersions in PBS (pH 6.25, ionic strength $150 \mathrm{mM}$ ) were applied in the QCM-D chamber, and changes in frequency $(\Delta f)$ and energy dissipation factor $(\Delta D)$ were simultaneously recorded. All experiments were conducted at $37{ }^{\circ} \mathrm{C}$ and at a constant flow rate of $50 \mu \mathrm{L} / \mathrm{min}$. QCM-D measurements were performed at in least triplicate, and representative measurements are presented.

\section{Exposure of biofilm to fluorescent and antimicrobial nanoparticles in a flow chamber system:}

Cultivation. Biofilms of $P$. aeruginosa were grown in a flow-chamber system containing individual 
channels with the dimensions of $1 \times 4 \times 40 \mathrm{~mm}$ as described previously ${ }^{41}$. Each channel of the flowchamber was inoculated with $300 \mu \mathrm{L}$ aliquots of overnight cultures of P. aeruginosa (PAO1 tagged with GFP), which were pre-grown in LB medium at $37{ }^{\circ} \mathrm{C}$ under vigorous shaking and adjusted to an optical density of 0.001 at $600 \mathrm{~nm}$ by using $0.9 \% \mathrm{NaCl}$ solution. After inoculation, the flow channels were left without flow for $1 \mathrm{~h}$ at $37^{\circ} \mathrm{C}$ to allow adhension of the bacterial cells to the substratum, after which the growth medium with $0.3 \mathrm{mM}$ glucose (SI, Table S2) was supplied at a flow rate of $3 \mathrm{~mL} / \mathrm{h}$ by using a peristaltic pump (Watson-Marlow 205S, Watson-Marlow, Cornwall, UK). The flowchamber system was incubated at $37^{\circ} \mathrm{C}$ for $3-4$ days until the biofilms were ready for the following investigation.

Nanoparticle penetration. To investigate the penetration of nanoparticles into the biofilms cultivated in the flow-chamber channels, each channel of the flow-chamber system was gently dosed with $300 \mu \mathrm{L}$ aliquots of fluorescent nanoparticles (DiIC18(3)-labeled PLGA nanoparticles and DiIC18(3)-labeled TPGS-PLGA hybrid nanoparticles), after which the system was left without flow for 30 min at $37{ }^{\circ} \mathrm{C}$. Subsequently, the $3 \mathrm{~mL} / \mathrm{h}$ flow of the growth medium with $0.3 \mathrm{mM}$ glucose (SI, Table S2) was resumed. The penetration of fluorescent nanoparticles into the biofilms was observed at pre-determined time intervals. To quantify the nanoparticles residing within the bacterial biofilm, the CLSM images (confocal z-stack) were first split into a bacterial channel and a nanoparticles channel. Subsequently, applying a gaussian smoothing factor to each slice, a mask was created using a hard threshold of 1.5 times the standard deviation of the image. This mask was applied to the nanoparticle channel to extract signal and hence avoid nonspecifically bound nanoparticles. Additionally, the mask allowed us to correct for the total amount of biofilm measured and hence provide normalized and comparable data between nanoparticles in an otherwise heterogeneous system. 
Killing efficacy. To investigate the killing efficacy of the antimicrobial nanoparticles (AZI-loaded TPGS-PLGA hybrid nanoparticles) against the biofilms, each channel was gently dosed with $300 \mu \mathrm{L}$ aliquots of antimicrobial nanoparticles (corresponding to a total of $1 \mathrm{mg} / \mathrm{mL}$ of AZI) and the system equilibrated as described above. To differentiate dead and live cells, propidium iodide (dead cell indicator) was added to the medium at a final concentration of $0.3 \mathrm{mM}{ }^{42}$. The dead and live cells in the biofilms were visualized at pre-determined time intervals. To quantify the amount of dead bacteria present in the bacterial biofilm, the following method was applied. Each image was split in a living biofilm channel (green) and a channel containing the dead bacteria (red), respectively. To ensure correct quantification and comparison of killed bacteria residing in the biofilm, a mask was created using the signal from both live and dead biofilm combined. The mask was created by applying sequentially to each slice in the z-stack a) a gaussian smoothing factor and b) hard threshold of 1.5 times the standard deviation of the given slice. By using the total integrated signal from the dead bacteria (red channel), and normalizing to the amount of biofilm measured, we were able to provide a relative killing efficiency difference between investigated nanoparticles. The killing efficiency was defined as follows:

$\mathrm{K}_{\mathrm{e}}=\frac{1}{\mathrm{n}} \sum_{0}^{\mathrm{n}}\left(\frac{1}{\mathrm{~V}_{\mathrm{B}, \mathrm{n}}} * \sum_{0}^{\mathrm{m}} \mathrm{S}_{\mathrm{D}, \mathrm{m}}\right)$

Where $K_{\mathrm{e}}$ is the killing efficiency corrected for amount of biofilm measured, $n$ is the number of images in a given experiment, $V_{\mathrm{B}}$ is the total volume of biofilm in image $n, \mathrm{~m}$ is the number of z-stacks in $\mathrm{n}$ and $S_{\mathrm{D}}$ is the signal from the dead bacterial biofilm in slice $m$.

Visualization. The biofilms, nanoparticle penetration and killing efficiency were visualized by using a confocal laser scanning microscope (Zeiss LSM 510, Carl Zeiss, Germany) equipped with detectors 
and filter sets for monitoring of GFP (excitation, $488 \mathrm{~nm}$; emission, $517 \mathrm{~nm}$ ), DiIC (18(3) $_{\text {and propidium }}$ iodide (excitation, $543 \mathrm{~nm}$; emission, $565 \mathrm{~nm}$ ). Images were obtained using a 40×/1.3 Plan-Neofluar oil immersion objective. Simulated 3D images and multichannel cross sections were generated using the Imaris software package (version 9.2) (Bitplane, Zürich, Switzerland).

\section{Results and discussion}

\section{Nanoparticle preparation and characterization}

TPGS-PLGA hybrid nanoparticles were synthesized via microvortex-induced rapid mixing of a PLGA organic solution in the inner channel with an aqueous solution containing TPGS in the outer channels by using a three-inlet microfluidic chip as described previously ${ }^{30}$. The use of microvortices enables up to 1000 times higher productivity than the conventional microfluidic methods based on diffusive mixing and convective mixing, thus, facilitates good manufacturing practice (GMP) production and clinical translation of nanoparticle-based formulations ${ }^{30}$. The microvortex pattern can be regulated by varying the Reynolds number $(R e)$ and the flow rate ratio of the inner organic solution to the outer aqueous solution. The previous studies demonstrated that the flow rate ratio of 1:10 and the $\operatorname{Re}$ of 150 can ensure high performance of nanoparticle synthesis ${ }^{43-44}$. Hence, these parameters were used in the present study for the synthesis of TPGS-PLGA hybrid nanoparticles, and resulted in the formation of nanoparticles with a size of less than $100 \mathrm{~nm}$ (Figure 1 and SI, Table S3). Langer and co-workers have previously shown that the formation of lipid-PLGA hybrid nanoparticles in the microfluidic-based onestep method indeed involves two distinct stages occurring within a very small time scale: $i$ ) formation of a PLGA core via the self-assembly of PLGA molecules owing to the solvents displacement; ii) lipid 
shell formation as soon as the lipid molecules assemble to the surface of the formed PLGA cores ${ }^{44}$. To investigate the role of TPGS on self-assembly of the nanoparticles, we investigated to what extent the self-assembly of PLGA was affected by the presence of TPGS. The increase in the concentration of TPGS from $0.1 \mathrm{mg} / \mathrm{mL}$ to $0.5 \mathrm{mg} / \mathrm{mL}$ decreased the nanoparticle size from $81 \mathrm{~nm}$ to $67 \mathrm{~nm}$. However, a further increase in the concentration of TPGS to $2 \mathrm{mg} / \mathrm{mL}$ resulted in an increased size, which could be because the elevated viscosity of the inner aqueous solution with the increasing TPGS concentration decreased the mixing efficiency. Additionally, the increase in the concentration of TPGS led to less negative zeta potentials from $-48 \mathrm{mV}$ to $-28 \mathrm{mV}$, which is indicative of the self-assembly of TPGS molecules on the surface of nanoparticles. The fluorescent nanoparticles with comparable sizes and zeta potentials were obtained by incorporating Atto655-conjugated PLGA or DiIC ${ }_{18(3)}$ into PLGA nanoparticles and TPGS-PLGA hybrid nanoparticles (SI, Table S3). Interestingly, it was observed that the encapsulation of AZI into the nanoparticles resulted in a smaller nanoparticle size (SI, Table S4), which is supported by the transmission electron microscopy (TEM) images (Figure 1c), though the mechanism is still unclear. In general, an average EE\% of AZI between 14\%-24\% was obtained (SI, Table S4), and can be slightly improved by increasing the $\mathrm{pH}$ of the aqueous phase due to the $\mathrm{pH}-$ dependent solubility of AZI ${ }^{45}$. The increase in the $\mathrm{pH}$ of the aqueous solution seemingly decreased the particle size, which could be attributed to the change in $\mathrm{pH}$ enhances the intermolecular interaction between AZI and the PLGA (e.g. hydrophobic interaction) during the assembly of the nanoparticles and shrink the dimension of the nanoparticles. The difference in zeta potentials induced by the $\mathrm{pH}$ of the aqueous solution could be due to the different surface enrichment of AZI and the different orientation of PLGA chains in the nanoparticles. However, no effects on size and zeta potential were observed with increasing the TPGS concentration in the aqueous phase from $1 \mathrm{mg} / \mathrm{mL}$ to $2 \mathrm{mg} / \mathrm{mL}$. 

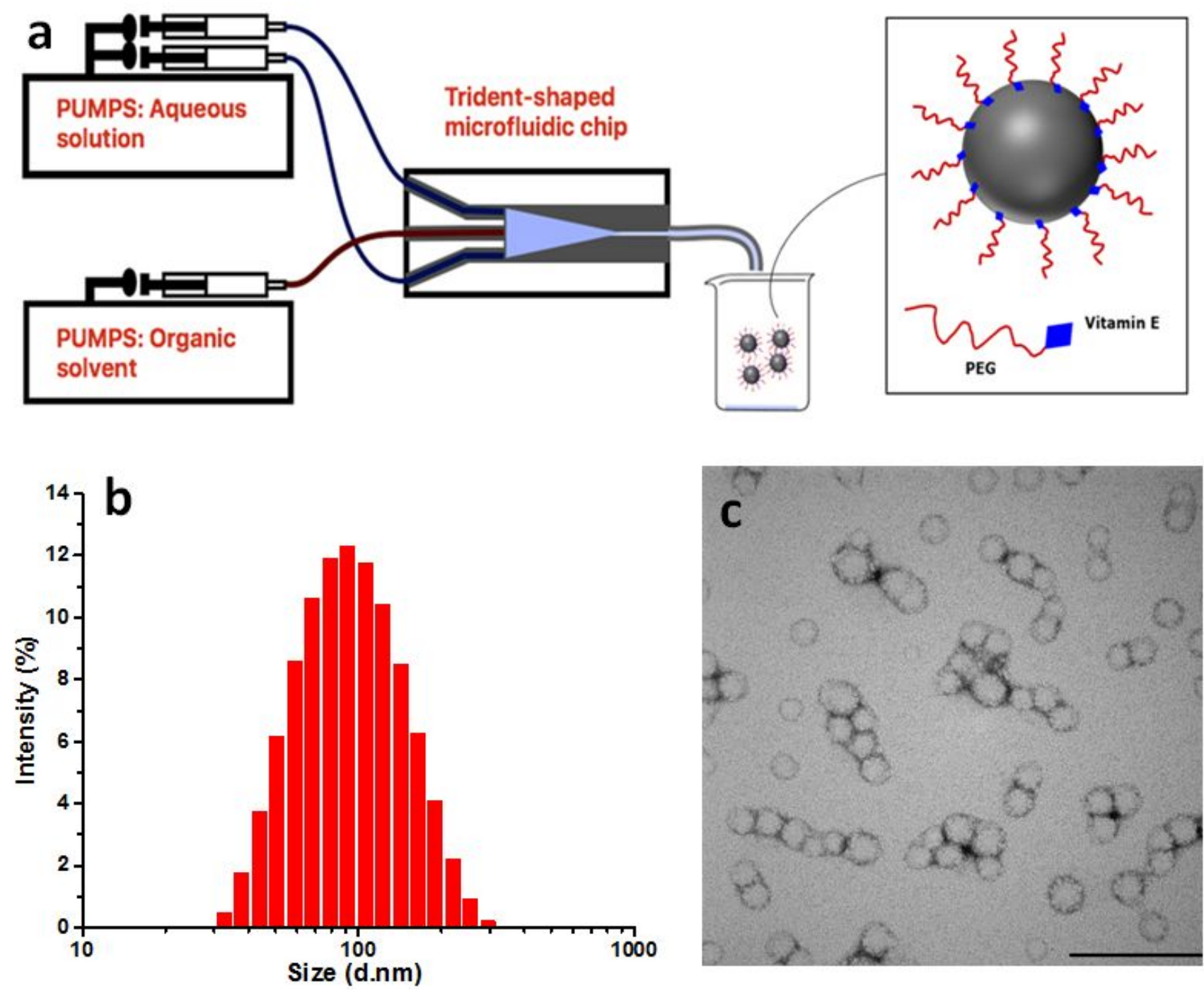

Figure 1. a) Schematic illustration of preparation and structure of TPGS-PLGA hybrid nanoparticles prepared by using the microfluidic method; b) Representative size distribution by intensity of TPGS-PLGA hybrid nanoparticles (type F, Table S3); c) Transmission electron microscopy (TEM) image of AZI-loaded TPGS-PLGA hybrid nanoparticles (type D, Table S4). Scale bar: $200 \mathrm{~nm}$.

\section{Penetration of nanoparticles in mucus}

The pathologically thick and viscous mucus layer lining the airways represents one of the important barriers to efficient antimicrobial therapy because the inhaled nanoparticles may be trapped via steric 
a

e
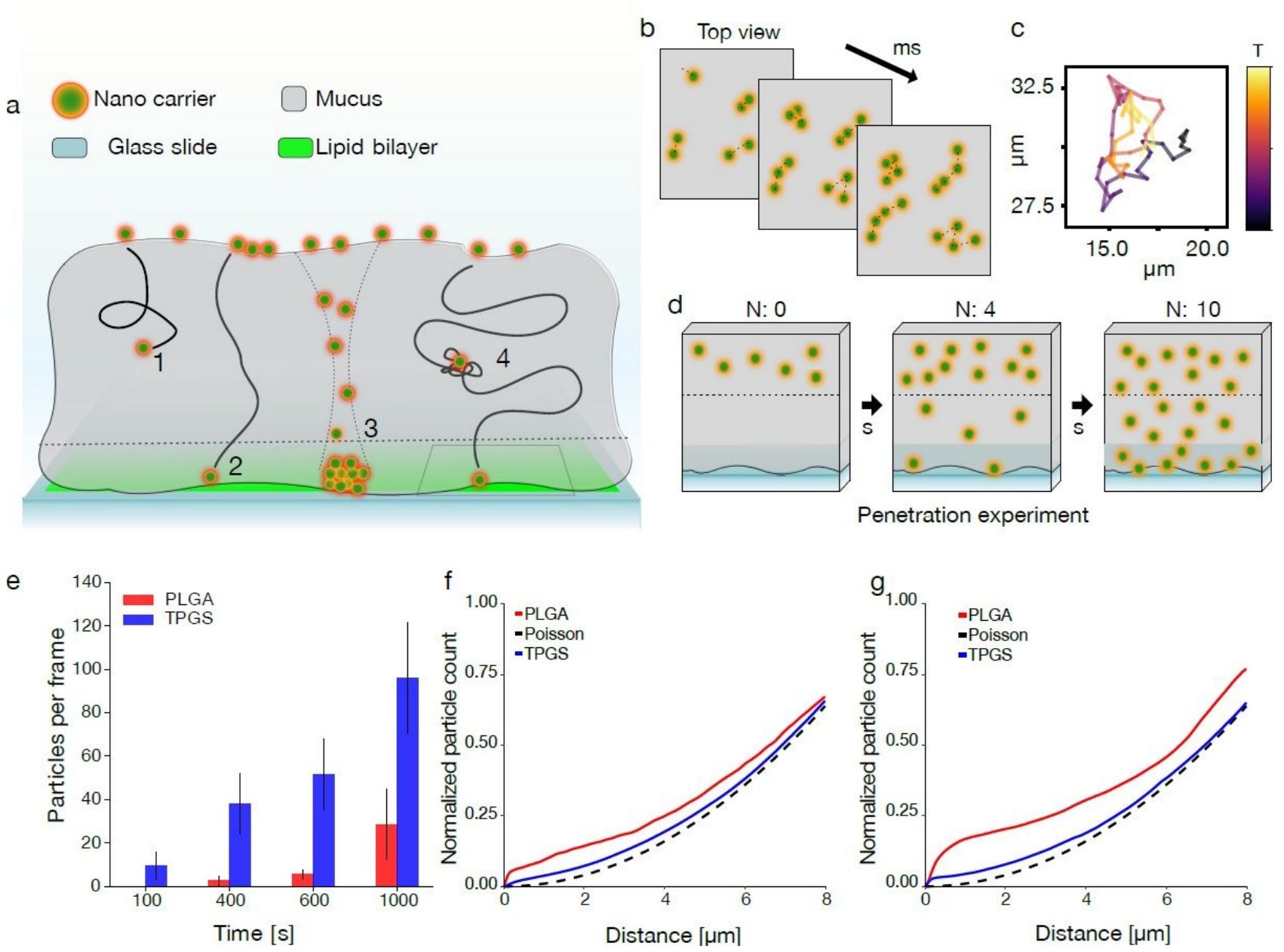

immobilization and/or adhesive interaction with the components in the mucus. In both cases, the restrained diffusion of the nanoparticles in the mucus will decrease their accumulation in close vicinity of the bacterial cells, and result in lower efficiency ${ }^{3,10}$. Thus, for successful delivery of antimicrobials to bacteria residing inside mucus, nanoparticles must be able to penetrate through mucus without being entrapped in the dense mucus network.

Figure 2. a) Cartoon illustration of nanoparticles penetrating through a mucosal layer placed on top of a microscopy glass slide and visualized by using total internal reflection fluorescence microscopy (TIRFM). Dashed line indicates observable zone, determined by the penetration depth used during acquisition $(200 \mathrm{~nm})$. Number 1, 2, 3, and 4 represent the distinct behaviors of nanoparticles in the mucus. Only the nanoparticles enter in the observable zone can be analyzed; b) Schematic illustration of TIRFM-based particle tracking with high temporal resolution allowing linking of distinct particles in time (tracking experimental setup); c) Representative moving trajectory of a single nanoparticle. Color code indicates observation time; d) Schematic illustration of TIRFM-based particle tracking with a low frame rate allowing long term measurements $(>40 \mathrm{~min})$ of the total number of nanoparticles per frame (penetration experimental setup); e) Time-dependent number of detected particles per frame (as displayed in d); $f$ ) 


\section{Spatial localization pattern of nanoparticles after $20 \mathrm{~min}$; g) Spatial localization pattern of nanoparticles after 40 min. Red: PLGA nanoparticles (type I, Table S3); blue: TPGS-PLGA hybrid nanoparticles (type J, Table S3).}

The mucus-penetrating property of the nanoparticles was investigated by using TIRFM-based particle tracking technique. Here, we applied the principle of TIRFM-based particle tracking to record the appearance of nanoparticles, which have penetrated the mucus layer and reached the evanescent plane of $200 \mathrm{~nm}$ (Figure 2a-d). Thus, compared to the conventional particle tracking, this approach allows for the exclusive detection of particle penetration through a biologically relevant thickness of mucus. Direct counting of the number of particles per frame showed a time-dependent increase for both the PLGA and the TPGS-PLGA hybrid nanoparticles, reflecting the process of nanoparticle penetrating across the mucus layer. Interestingly, the TPGS-PLGA hybrid nanoparticles presented a faster onset of penetration and a steeper slope of the particle number-time curve (Figure 2e) during the initial $20 \mathrm{~min}$, indicative of a more superior mucus-penetrating property compared to PLGA nanoparticles. In addition, the PLGA nanoparticles showed evident penetration, which could be attributed to the ultrasmall size of PLGA nanoparticles. As reported previously, the mesh spacing of fresh, undiluted and unaltered CF sputum ranges $60-300 \mathrm{~nm}{ }^{15}$. Thus, for the nanoparticles with the diameter less than 60 $\mathrm{nm}$, the steric hindrance of mucus would be minimized. The difference in mucus-penetrating behaviors between PLGA nanoparticles and TPGS-PLGA hybrid nanoparticles could mainly result from their adhesive interaction with mucus. Thus, to decipher the interaction of the nanoparticles with the mucus, a spatial analysis of particle density was conducted by using the Ripley's K function ${ }^{37}$, which counts the average distance from a particle to its neighbors (SI, Figure S4), thus, allowing one to quantitatively describe the degree of nanoparticles localization at the certain areas due to such bio-nano interaction. The spatial localization pattern showed a random distribution pattern of TPGS-PLGA hybrid nanoparticles, suggesting that TPGS-PLGA hybrid nanoparticles can effectively 'slip' through the 

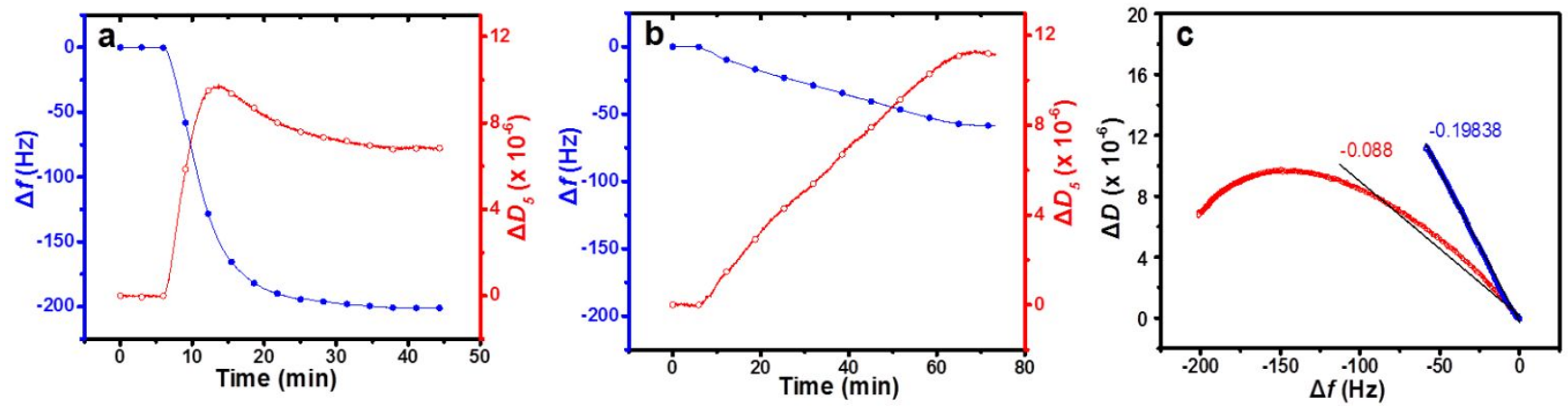

Figure 3. a) Representative QCM-D measurements of the changes in frequency ( $\Delta f$, blue) and energy dissipation factor $(\Delta D$, red) upon the interaction of PLGA nanoparticles (type A, Table S3) with a mucin layer at pH 6.25, ionic strength $150 \mathrm{mM}$; b) TPGS-PLGA hybrid nanoparticles (type F, Table S3) with a mucin layer at pH 6.25, ionic strength $150 \mathrm{mM}$; c) $\Delta D-\Delta f$ plots of adsorption of PLGA nanoparticles (red) and TPGS-PLGA nanoparticles (blue) onto a mucin layer at pH6.25, ionic strength $150 \mathrm{mM}$.

QCM-D measurements were further performed to evaluate the adhesive interactions between mucin and nanoparticles, as previously reported ${ }^{46}$. Introduction of the PLGA nanoparticles onto the preformed mucin layer representing a pathologically relevant lung condition $(\mathrm{pH} 6.25$, ionic strength $150 \mathrm{mM}$ ) resulted in a $\Delta f$ of about $-200 \mathrm{~Hz}$ (for the fifth harmonic overtone, i.e., $\mathrm{n}=5$ ) (Figure 3a). In comparison, introduction of the TPGS-PLGA hybrid nanoparticles onto the mucin layer representing the identical pathologically relevant lung condition ( $\mathrm{pH} 6.25$, ionic strength $150 \mathrm{mM}$ ) led to a $\Delta f$ of -60 $\mathrm{Hz}$ in 60 min (Figure 3b), suggesting much slower dynamics of adsorption of the TPGS-PLGA hybrid nanoparticles onto the mucin layer. Additionally, it was observed that the $\Delta D$ versus $\Delta f$ plot of the PLGA nanoparticles showed a less steep slope than that obtained for the TPGS-PLGA hybrid nanoparticles (Figure 3c), indicating that the TPGS coating effectively reduced the stiffness of the interaction between nanoparticle and mucin. This would be attributed to the hydrophilic and less 
negatively charged surface of the TPGS-PLGA hybrid nanoparticles. Interestingly, upon introducing the PLGA nanoparticles onto the immobilized mucin layer, $\Delta D$ increased promptly to $\sim 10 \times 10^{-6}$ at the initial stage (within first $10 \mathrm{~min}$ ). However, a gentle decline of $\Delta D$ (to $\sim 6 \times 10^{-6}$ ) was observed with the continuous adsorption of the PLGA nanoparticles (Figure 3a). This phenomenon could result from the transformation of a 'soft' mucin layer to a more 'rigid' layer, which is likely induced by the intensive adhesive interaction between the PLGA nanoparticles and the mucin layer. In line with the particle tracking results, these observations suggest that the TPGS coating can effectively reduce the mucinnanoparticle interaction and improve the mucus-penetrating property of the nanoparticles.

\section{Accumulation of nanoparticles in bacterial biofilms}

The spatial location of the fluorescent nanoparticles in P. aeruginosa biofilms grown in a flowchamber system under well-controlled and hydrodynamic conditions was visualized by using confocal laser scanning microscopy (CLSM) ${ }^{41}$. This flow-chamber system allows for visualization of the complex multicellular structures present in P. aeruginosa biofilms (e.g., mushroom-shaped biofilms) ${ }^{47}$, and observation of nanoparticle penetration into the biofilms. In addition, the nanoparticles that do not bind to the bacterial biofilms will be removed with the hydrodynamic flow in the chambers, which is a highly relevant to an in vitro lung clearance model. This is highly advantageous compared to the conventional methodologies applying static conditions, since more dynamic conditions will include the effects of shear stress and clearance. 

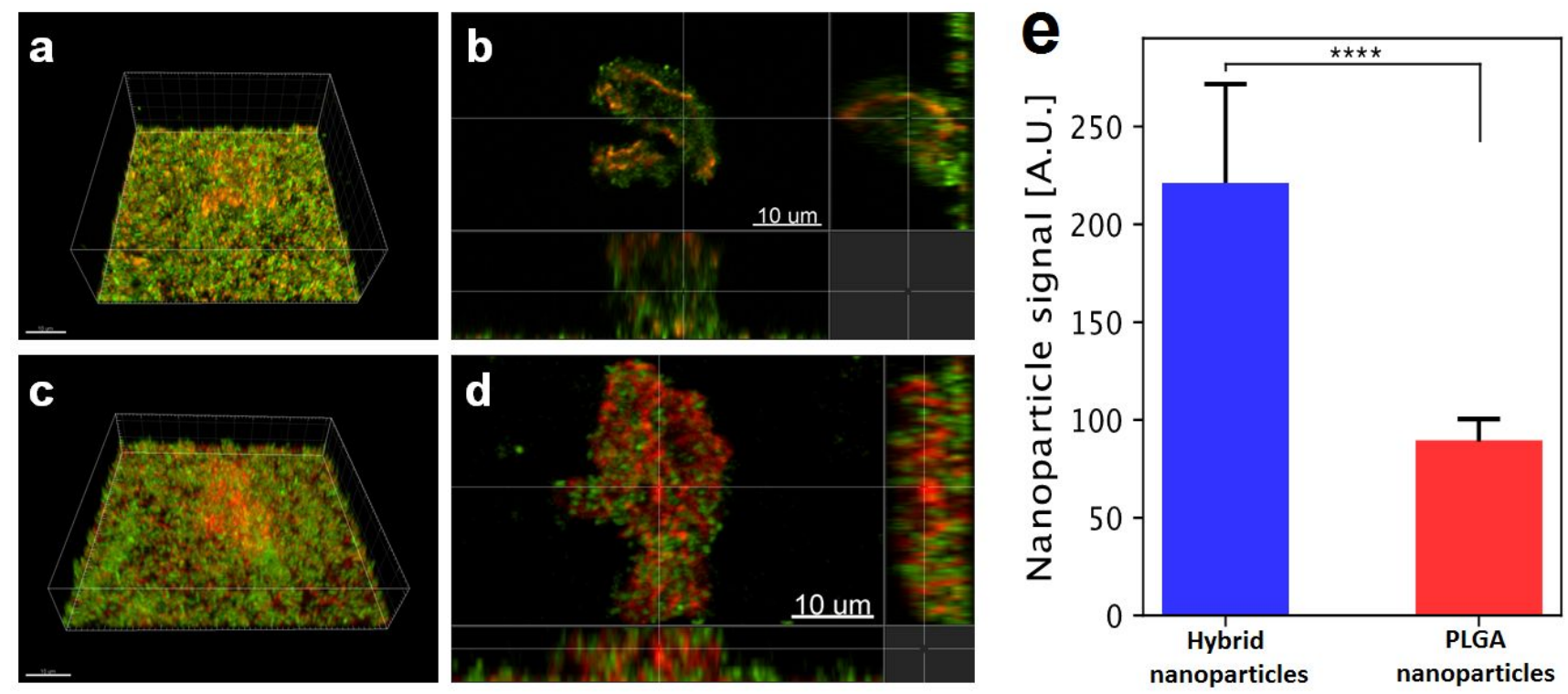

Figure 4. Penetration of DiIC ${ }_{18(3)}$-labeled PLGA nanoparticles (type G, Table S3) and DiIC $_{18(3)}$-labeled TPGS-PLGA hybrid nanoparticles (type $\mathrm{H}$, Table S3) in P. aeruginosa biofilm (PAO1 tagged with GFP). a) and b) CLSM images of DiIC ${ }_{18(3)}$-labeled PLGA nanoparticles accumulated in $P$. aeruginosa biofilm at $5 \mathrm{~h}$. c) and d) CLSM images of DiIC $_{18(3)}$-labeled TPGS-PLGA hybrid nanoparticles accumulated in $P$. aeruginosa biofilm at $5 \mathrm{~h}$. a) and c): 3D images; b) and d): 2D cross-section images (the central pictures show top-down views, and the flanking pictures show side views). P. aeruginosa bacteria appear green due to expression of GFP and the fluorescent nanoparticles appear red due to labeling with DiIC $_{18(3)}$. Scale bars: $10 \mu \mathrm{m}$. e) Quantitative analysis of nanoparticles in bacterial biofilm. Statistics by students t-test: **** indicate p-value $<0.0001$.

Exposure of the heterogeneous multicellular $P$. aeruginosa biofilms with both $\mathrm{DiIC}_{18(3)}$-labeled PLGA nanoparticles and DiIC ${ }_{18(3)}$-labeled TPGS-PLGA hybrid nanoparticles resulted in accumulation of redfluorescent nanoparticles in green-fluorescent (GFP-tagged) P. aeruginosa biofilms (Figure 4a, c), indicating adequate penetration of both nanoparticles into the bacterial biofilm due to their ultra-small size (less than $100 \mathrm{~nm}$, SI, Table S3). However, the fluorescent PLGA nanoparticles appeared to primarily localize at the superficial layer of the biofilm (Figure 4b). In contrast, the fluorescent TPGSPLGA hybrid nanoparticles were able to penetrate deeper into the central part of the biofilm (Figure 4d), suggesting superior biofilm-penetrating property of the TPGS-PLGA hybrid nanoparticles. Quantitative imaging analysis of the penetration of nanoparticles in biofilms displayed that the TPGSPLGA hybrid nanoparticles presented approximately 2 times higher density in the bacterial biofilms 
compared to PLGA nanoparticles (Figure 4e). The observation indicates that the surface-enriched carboxyl groups of PLGA nanoparticles may strongly interact with and bind to the components of the EPS, thus, hindering the diffusion of PLGA nanoparticles into the deeper layer of the biofilms. In contrast, the surface-enriched PEG moieties of TPGS-PLGA hybrid nanoparticles effectively display reduced interaction and binding of the nanoparticles with the components of the EPS, thus, allowing for adequate penetration of the nanoparticles into the deeper layer of the biofilms.
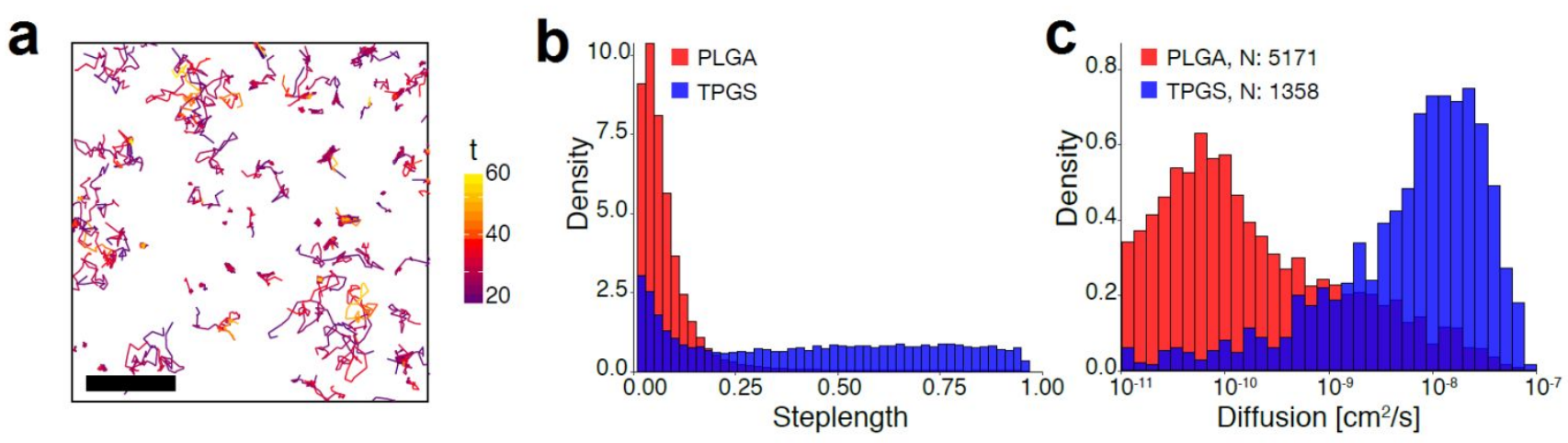

Figure 5. a) Representative moving trajectories of nanoparticles at the boundary between the mucus and the bacterial outer membrane-mimicking lipid bilayer obtained from tracking experiments (showed in Figure $2 \mathrm{~b}$, color code indicates observation time); b) Distribution of the step size; c): Distributions of the effective diffusivities $\left(D_{\text {eff }}\right)$. Red: PLGA nanoparticles (type I, Table S3); Blue: TPGS-PLGA hybrid nanoparticles (type J, Table S3).

The mobility of the nanoparticles at the interface between mucus and the bacterial membrane was investigated by using TIRFM-based particle tracking with the tracking experimental setup (Figure 2b). The glass slides in a microscopy chamber were coated with a lipid bilayer composed of POPE:POPG=3:1. This lipid mixture mimics the charges of the outer membrane of P. aeruginosa ${ }^{33}$ and is ideal for in vitro quantitative analysis of the interaction, kinetics and diffusional properties of the two nanoparticle systems at the interface between mucus and the bacterial membrane. The high fidelity of the assay allowed for identification and tracking of hundreds of individual nanoparticles per frame (>1000) simultaneously within a single experiment (Figure 5a), and estimation of diffusion coefficient 
and individual step sizes (i.e. the displacements in position of nanoparticles between frames). Compared to PLGA nanoparticles, the TPGS-PLGA hybrid nanoparticles presented up to 5 times longer step lengths with a more heterogeneous distribution (Figure 5b) and a higher diffusion coefficient (Figure 5c), indicating the higher mobility of the TPGS-PLGA hybrid nanoparticles at the boundary between the mucus and the bacterial outer membrane-mimicking lipid bilayer. This higher mobility could, however, potentially facilitate the escape of TPGS-PLGA hybrid nanoparticles from the biofilm with the hydrodynamic flow, thus, could represent a drawback to the long-term sustained delivery of antimicrobials in the biofilms ${ }^{12}$. However, it was observed that TPGS-PLGA hybrid nanoparticles presented a comparable retention in biofilm after $24 \mathrm{~h}$ with that of PLGA nanoparticles (SI, Figure S5), though both nanoparticles demonstrated decreased accumulation in the biofilm at $24 \mathrm{~h}$, suggesting that TPGS coating did not promote the escape of nanoparticles from the biofilm. This observation indirectly supports our expectation that TPGS could be cleaved by esterase produced by $P$. aeruginosa bacteria, thus leading to the surface-switching property of TPGS-PLGA hybrid nanoparticles and allowing the 'decayed' nanoparticles for anchoring in the biofilm.

In summary, the TPGS-PLGA hybrid nanoparticles present hydrophilic surfaces with slight negative charges, which offer superior ability to penetrate through mucus and into the deeper layer of bacterial biofilms. Once localized in the biofilms, and the TPGS shell of the nanoparticles is cleaved into PEG and vitamin E, resulting in a lipophilic vitamin E-enriched surface of nanoparticles, this further promotes accumulation of nanoparticles in the biofilms.

\section{Killing efficacy against biofilm-forming $P$. aeruginosa}


The therapeutic efficacy of AZI in CF patients has been proven in many clinical trials ${ }^{48-50}$, though it is not yet approved for the treatment of CF. In light of the promising findings, we employed AZI as an antimicrobial agent to investigate the anti-biofilm efficacy of AZI-loaded TPGS-PLGA hybrid nanoparticles against CF-related $P$. aeruginosa biofilms. The minimum inhibitory concentration (MIC) of AZI in solution for planktonic P. aeruginosa was determined to be $512 \mu \mathrm{g} / \mathrm{mL}$, which is consistent with the early reports ${ }^{29}$. In our previous study, encapsulation of AZI in TPGS-PLGA hybrid nanoparticles increased effect of AZI on prevention and eradication of $P$. aeruginosa biofilms due to inhibition of quorum sensing-associated virulence factors (e.g. decrease the bacterial swarming motility and the production of pyocyanin and pyoverdin) ${ }^{51}$.
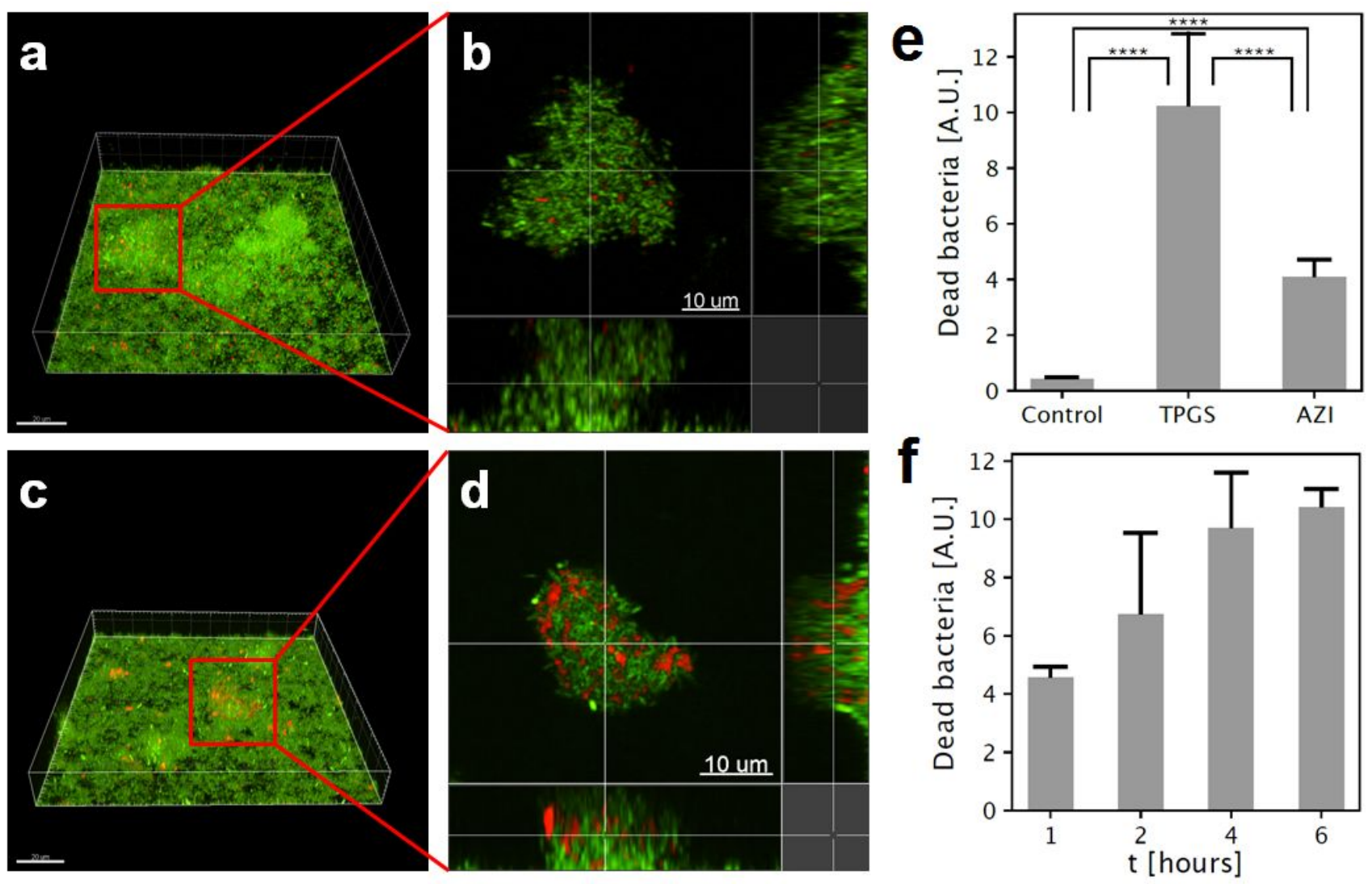


\begin{abstract}
Figure 6. Killing efficacy of AZI and AZI-loaded TPGS-PLGA hybrid nanoparticles (type D, Table S4) against $P$. aeruginosa biofilm (PAO1 tagged with GFP). The alive and dead $P$. aeruginosa bacteria appear green and red, respectively. a) and b) CLSM images of $P$. aeruginosa biofilm treated with AZI at 6 h. c) and d) CLSM images of $P$. aeruginosa biofilm treated with AZI-loaded TPGS-PLGA hybrid nanoparticles at 6 h. The alive and dead $P$. aeruginosa bacteria appear green and red, respectively. Scale bars: $10 \mu \mathrm{m}$. e) Quantification of relative killing efficiency of AZI and AZI-loaded TPGS-PLGA hybrid nanoparticles against $P$. aeruginosa biofilm. Control group: $P$. aeruginosa biofilm was only treated with PI. f) Time dependency of killing efficiency of AZI-loaded TPGS-PLGA hybrid nanoparticles. Error bars indicate error of the mean (N=3, 14 and 11 for control, TPGS, and AZI, respectively). Statistics by students t-test: $* * * *$ indicate p-value $<\mathbf{0 . 0 0 0 1}$.
\end{abstract}

The nanoparticle-mediated killing efficacy against biofilm-forming $P$. aeruginosa was investigated by using the flow-chamber system by comprising the effects of shear stress and clearance. P. aeruginosa biofilms preformed in the flow-chamber system were treated with AZI and AZI-loaded TPGS-PLGA hybrid nanoparticles, followed by staining with propidium iodide (PI, specifically stain the dead bacterial cells ${ }^{52}$ ). The killing efficacy was evaluated by using the fluorescent intensity (red color) of dead bacterial cells in the CLSM images. As observed in CLSM images, $P$. aeruginosa biofilms treated with AZI-loaded TPGS-PLGA hybrid nanoparticles presented more dead bacterial cells (red) than those treated with AZI (Figure 6a-d). Quantitative analysis of CLSM images demonstrated that AZIloaded TPGS-PLGA hybrid nanoparticles presented approximately 2.5 time higher killing efficacy than AZI at $6 \mathrm{~h}$ (Figure 6e). Meanwhile, the effect of PI on the bacterial biofilms was only subtle within the investigating time $(6 \mathrm{~h})$. The enhanced killing efficacy could mainly attribute to that TPGS-PLGA hybrid nanoparticles promote the site-specific delivery of AZI and increase the drug concentration in the close proximity of the biofilm-forming bacteria. In addition, it should be noted that Vitamin E released from the enzymatic hydrolysis of TPGS, as a well-known antioxidant, could also contribute to improving the susceptibility of $P$. aeruginosa to antimicrobial therapeutics and delaying the development of antimicrobial resistance ${ }^{53-54}$. In addition, AZI nanoparticles formulation displayed a time-dependent killing efficacy (Figure 6f). This time-dependent killing efficacy could result from the sustained release behavior of the AZI-loaded nanoparticles formulation (SI, Figure S6) ${ }^{55}$. It is worth to 
mention that, in previous studies, the beneficial effects on CF patients treated with AZI in solution have been demonstrated only up to 6 months, while longer durations of treatment resulted in reduced efficacy probably due to the emergence of antibiotic tolerant $P$. aeruginosa subpopulations ${ }^{29,56}$. The sustained drug release from the TPGS-PLGA hybrid nanoparticles can provide a steady drug pharmacokinetic/pharmacodynamic profile for prolonged period, thus, may represent a promising approach to prevent the emergence of antibiotic tolerant $P$. aeruginosa subpopulations ${ }^{3,57}$.

\section{Conclusion}

In conclusion, we demonstrate that ultra-small TPGS-PLGA hybrid nanoparticles can potentially adapt to the complicated pathological environment in lungs of CF patients and achieve the site-specific delivery of antibiotics into bacterial biofilms. The TPGS-PLGA hybrid nanoparticles present the superior ability to penetrate through the mucus and accumulate into the interior of $P$. aeruginosa biofilms. As a consequence, encapsulation of AZI into TPGS-PLGA hybrid nanoparticles promotes the killing efficacy against biofilm-forming $P$. aeruginosa bacteria with a time-dependent manner. We, therefore, propose that TPGS-PLGA hybrid antimicrobial nanoparticles could improve the treatment of CF-related bacterial biofilm infections.

Supporting Information. Synthesis and characterization of Atto655-conjugated PLGA; high performance liquid chromatography with evaporative light scattering detector (ELSD-HPLC); schematic representation of the Ripley's K function used for spatial point patterns; CLSM images of $P$. aeruginosa (PAO1) biofilms treated with $\mathrm{DiIC}_{18(3)}$-labeled nanoparticles; in vitro release profile of 
AZI from AZI-loaded TPGS-PLGA hybrid nanoparticles; composition of the growth medium for $P$. aeruginosa biofilm cultured in the flow-chamber system; preparation parameters, size, PDI, and zeta potential of PLGA nanoparticles, TPGS-PLGA hybrid nanoparticles, and fluorescent nanoparticles; preparation and characterization of AZI-loaded TPGS-PLGA hybrid nanoparticles.

\section{Acknowledgements}

This work was funded by the Danish Council for Independent Research, Technology and Production Sciences (DFF-4093-00062) (FW), Lundbeck Foundation (R264-2017-3404) (FW), and the University of Copenhagen 2016 Programme of Excellence Research Centre for Control of Antibiotic Resistance (UC-CARE) (SNK). HMN acknowledges funding from Novo Nordisk Foundation (Grand Challenge Program; NNF16OC0021948). NSH acknowledges funding from Villum Young Investigator program (grant 10099) and the Carlsberg foundation distinguished Associate Professor Program (CF16-0797).

\section{References}

(1) Govan, J. R.; Deretic, V. Microbial Pathogenesis in Cystic Fibrosis: Mucoid Pseudomonas Aeruginosa and Burkholderia Cepacia. Microbiol. Mol. Biol. Rev. 1996, 60 (3), 539-574.

(2) Lechtzin, N.; John, M.; Irizarry, R.; Merlo, C.; Diette, G. B.; Boyle, M. P. Outcomes of Adults with Cystic Fibrosis infected with Antibiotic-resistant Pseudomonas Aeruginosa. Respiration 2006, 73 (1), 27-33, DOI: $10.1159 / 000087686$.

(3) Ciofu, O.; Tolker-Nielsen, T.; Jensen, P. O.; Wang, H.; Hoiby, N. Antimicrobial Resistance, Respiratory Tract Infections and Role of Biofilms in Lung Infections in Cystic Fibrosis Patients. Adv. Drug Deliv. Rev. 2015, 85, 7-23, DOI: 10.1016/j.addr.2014.11.017.

(4) Geller, D. E.; Pitlick, W. H.; Nardella, P. A.; Tracewell, W. G.; Ramsey, B. W. Pharmacokinetics and Bioavailability of Aerosolized Tobramycin in Cystic Fibrosis. Chest 2002, 122 (1), 219-226, DOI: 10.1378/chest.122.1.219.

(5) Gibson, R. L.; Retsch-Bogart, G. Z.; Oermann, C.; Milla, C.; Pilewski, J.; Daines, C.; Ahrens, R.; Leon, K.; Cohen, M.; McNamara, S.; Callahan, T. L.; Markus, R.; Burns, J. L. Microbiology, Safety, and Pharmacokinetics of Aztreonam lysinate for Inhalation in Patients with Cystic Fibrosis. Pediatr. Pulmonol. 2006, 41 (7), 656-665, DOI: 10.1002/ppul.20429. 
(6) Dudley, M. N.; Loutit, J.; Griffith, D. C. Aerosol Antibiotics: Considerations in Pharmacological and Clinical evaluation. Curr. Opin. Biotechnol.2008, 19 (6), 637-643, DOI: 10.1016/j.copbio.2008.11.002.

(7) Fahy, J. V.; Dickey, B. F. Airway Mucus Function and Dysfunction. N. Engl. J. Med. 2010, 363 (10), 22332247.

(8) Treacy, K.; Tunney, M.; Elborn, J. S.; Bradley, J. M. Mucociliary Clearance in Cystic Fibrosis: Physiology and Pharmacological Treatments. Paediatr. Child Health 2011, 21 (9), 425-430, DOI:

10.1016/j.paed.2011.05.011.

(9) Flemming, H. C.; Wingender, J. The Biofilm Matrix. Nat. Rev. Microbiol. 2010, 8 (9), 623-633, DOI: $10.1038 /$ nrmicro2415.

(10) Costerton, J. W.; Stewart, P. S.; Greenberg, E. P. Bacterial Biofilms: A Common Cause of Persistent Infections. Science 1999, 284 (5418), 1318-1322.

(11) Huh, A. J.; Kwon, Y. J. "Nanoantibiotics": A New Paradigm for Treating Infectious Diseases using Nanomaterials in the Antibiotics Resistant Era. J. Control. Release 2011, 156 (2), 128-145, DOI:

10.1016/j.jconrel.2011.07.002.

(12) Forier, K.; Raemdonck, K.; De Smedt, S. C.; Demeester, J.; Coenye, T.; Braeckmans, K. Lipid and Polymer Nanoparticles for Drug Delivery to Bacterial Biofilms. J. Control. Release 2014, 190, 607-623.

(13) Garcia-Diaz, M.; Birch, D.; Wan, F.; Nielsen, H. M. The Role of Mucus as An Invisible Cloak to Transepithelial Drug Delivery by Nanoparticles. Adv. Drug Deliv. Rev.2018, 124, 107-124, DOI:

10.1016/j.addr.2017.11.002.

(14) Forier, K.; Messiaen, A. S.; Raemdonck, K.; Nelis, H.; De Smedt, S.; Demeester, J.; Coenye, T.; Braeckmans, K. Probing the Size Limit for Nanomedicine Penetration into Burkholderia Multivorans and Pseudomonas Aeruginosa Biofilms. J. Control. Release 2014, 195, 21-28, DOI: 10.1016/j.jconrel.2014.07.061. (15) Suk, J. S.; Lai, S. K.; Wang, Y. Y.; Ensign, L. M.; Zeitlin, P. L.; Boyle, M. P.; Hanes, J. The Penetration of Fresh Undiluted Sputum Expectorated by Cystic Fibrosis Patients by Non-adhesive Polymer Nanoparticles. Biomaterials 2009, 30 (13), 2591-2597, DOI: 10.1016/j.biomaterials.2008.12.076.

(16) Liu, Y.; Busscher, H. J.; Zhao, B.; Li, Y.; Zhang, Z.; van der Mei, H. C.; Ren, Y.; Shi, L. Surface-Adaptive, Antimicrobially Loaded, Micellar Nanocarriers with Enhanced Penetration and Killing Efficiency in Staphylococcal Biofilms. ACS nano 2016, 10 (4), 4779-4789, DOI: 10.1021/acsnano.6b01370.

(17) Radovic-Moreno, A. F.; Lu, T. K.; Puscasu, V. A.; Yoon, C. J.; Langer, R.; Farokhzad, O. C. Surface Charge-Switching Polymeric Nanoparticles for Bacterial Cell Wall-Targeted Delivery of Antibiotics. ACS nano 2012, 6 (5), 4279-4287.

(18) Horev, B.; Klein, M. I.; Hwang, G.; Li, Y.; Kim, D.; Koo, H.; Benoit, D. S. W. pH-activated Nanoparticles for Controlled Topical Delivery of Farnesol to Disrupt Oral Biofilm Virulence. ACS nano 2015, 9 (3), 23902404.

(19) Tate, S.; MacGregor, G.; Davis, M.; Innes, J.; Greening, A. Airways in Cystic Fibrosis are Acidified: Detection by Exhaled Breath Condensate. Thorax.2002, 57 (11), 926-929.

(20) Poschet, J.; Perkett, E.; Deretic, V. Hyperacidification in Cystic Fibrosis: Links with Lung Disease and New Prospects for Treatment. Trends Mol. Med. 2002, 8 (11), 512-519.

(21) Bodem, C. R.; Lampton, L. M.; Miller, D. P.; Tarka, E. F.; Everett, E. D. Endobronchial pH. Relevance of Aminoglycoside Activity in Gram-negative Bacillary Pneumonia. Am. Rev. Respir. Dis. 1983, 127 (1), 39-41.

(22) Smith, J. J.; Travis, S. M.; Greenberg, E. P.; Welsh, M. J. Cystic Fibrosis Airway Epithelia Fail to Kill Bacteria Because of Abnormal Airway Surface Fluid. Cell 1996, 85 (2), 229-236.

(23) Zhang, Z.; Tan, S.; Feng, S. S. Vitamin E TPGS as A Molecular Biomaterial for Drug Delivery.

Biomaterials 2012, 33 (19), 4889-4906, DOI: 10.1016/j.biomaterials.2012.03.046.

(24) Guo, Y.; Luo, J.; Tan, S.; Otieno, B. O.; Zhang, Z. The Applications of Vitamin E TPGS in Drug Delivery. Eur. J. Pharm. Sci. 2013, 49 (2), 175-186, DOI: 10.1016/j.ejps.2013.02.006.

(25) Dahe, G. J.; Kadam, S. S.; Sabale, S. S.; Kadam, D. P.; Sarkate, L. B.; Bellare, J. R. In vivo Evaluation of the Biocompatibility of Surface Modified Hemodialysis Polysulfone Hollow Fibers in Rat. PloS one 2011, 6 (10), e25236, DOI: 10.1371/journal.pone.0025236. 
(26) Tielen, P.; Rosenau, F.; Wilhelm, S.; Jaeger, K. E.; Flemming, H. C.; Wingender, J. Extracellular Enzymes Affect Biofilm Formation of Mucoid Pseudomonas Aeruginosa. Microbiology 2010, 156 (Pt 7), 2239-2252, DOI: 10.1099/mic.0.037036-0.

(27) Kozirog, A.; Otlewska, A.; Brycki, B. Viability, Enzymatic and Protein Profiles of Pseudomonas aeruginosa Biofilm and Planktonic Cells after Monomeric/Gemini Surfactant Treatment. Molecules 2018, 23 (6), E1294, DOI: 10.3390/molecules23061294.

(28) Lai, S. K.; Wang, Y. Y.; Hanes, J. Mucus-penetrating Nanoparticles for Drug and Gene Delivery to Mucosal Tissues. Adv. Drug Deliv. Rev. 2009, 61 (2), 158-171, DOI: 10.1016/j.addr.2008.11.002.

(29) Imperi, F.; Leoni, L.; Visca, P. Antivirulence Activity of Azithromycin in Pseudomonas Aeruginosa. Front. Microbiol.2014, 5, 178, DOI: 10.3389/fmicb.2014.00178.

(30) Kim, Y.; Lee Chung, B.; Ma, M.; Mulder, W. J.; Fayad, Z. A.; Farokhzad, O. C.; Langer, R. Mass Production and Size Control of Lipid-polymer Hybrid Nanoparticles through Controlled Microvortices. Nano Lett.2012, 12 (7), 3587-3591, DOI: 10.1021/n1301253v.

(31) Boegh, M.; Baldursdottir, S. G.; Mullertz, A.; Nielsen, H. M. Property Profiling of Biosimilar Mucus in a Novel Mucus-containing in vitro Model for Assessment of Intestinal Drug Absorption. Eur. J. Pharm.

Biopharm.e.V 2014, 87 (2), 227-235, DOI: 10.1016/j.ejpb.2014.01.001.

(32) Cremer, P. S.; Boxer, S. G. Formation and Spreading of Lipid Bilayers on Planar Glass Supports. J. Phys. Chem. B 1999, 103 (13), 2554-2559.

(33) Strömstedt, A. A.; Ringstad, L.; Schmidtchen, A.; Malmsten, M. Interaction Between Amphiphilic Peptides and Phospholipid Membranes. Curr. Opin. Colloid Interface Sci. 2010, 15 (6), 467-478, DOI:

10.1016/j.cocis.2010.05.006.

(34) Tinevez, J. Y.; Perry, N.; Schindelin, J.; Hoopes, G. M.; Reynolds, G. D.; Laplantine, E.; Bednarek, S. Y.; Shorte, S. L.; Eliceiri, K. W. TrackMate: An Open and Extensible Platform for Single-particle Tracking. Methods 2017, 115, 80-90, DOI: 10.1016/j.ymeth.2016.09.016.

(35) Lowe, D. G. Distinctive Image Features from Scale-Invariant Keypoints. Int. J. Comput. Vis. 2004, 60 (2), 91-110, DOI: 10.1023/B:VISI.0000029664.99615.94.

(36) Bretzner, L.; Lindeberg, T. Feature Tracking with Automatic Selection of Spatial Scales. Comput. Vis. Image Underst. 1998, 71 (3), 385-392, DOI: https://doi.org/10.1006/cviu.1998.0650.

(37) Ripley, B. D. The Second-Order Analysis of Stationary Point Processes. J. Appl. Probab. 1976, 13 (2), 255 266, DOI: $10.2307 / 3212829$.

(38) Konopka, M. C.; Weisshaar, J. C. Heterogeneous Motion of Secretory Vesicles in the Actin Cortex of Live Cells: 3D Tracking to 5-nm Accuracy. J. Phys. Chem. A 2004, 108 (45), 9814-9826.

(39) Rocha, S.; Hutchison, J. A.; Peneva, K.; Herrmann, A.; Mullen, K.; Skjot, M.; Jorgensen, C. I.; Svendsen, A.; De Schryver, F. C.; Hofkens, J.; Uji-i, H. Linking Phospholipase Mobility to Activity by Single-molecule Wide-field Microscopy. Chemphyschem 2009, 10 (1), 151-161, DOI: 10.1002/cphc.200800537.

(40) Dahan, M.; Le'vi, S.; Luccardini, C.; Rostaing, P.; Riveau, B. a.; Triller, A. Diffusion Dynamics of Glycine Receptors Revealed by Single-quantum Dot Tracking. Science 2003, 302 (5644), 442-445.

(41) Weiss Nielsen, M.; Sternberg, C.; Molin, S.; Regenberg, B. Pseudomonas Aeruginosa and Saccharomyces Cerevisiae Biofilm in Flow Cells. J. Vis. Exp. 2011, (47), pii: 2383, DOI: 10.3791/2383.

(42) Haagensen, J. A. J.; Klausen, M.; Ernst, R. K.; Miller, S. I.; Folkesson, A.; Tolker-Nielsen, T.; Molin, S. Differentiation and Distribution of Colistin- and Sodium Dodecyl Sulfate-Tolerant Cells in Pseudomonas aeruginosa Biofilms. J. Bacteriol.2006, 189 (1), 28-37, DOI: 10.1128/jb.00720-06.

(43) Kim, Y.; Fay, F.; Cormode, D. P.; Sanchez-Gaytan, B. L.; Tang, J.; Hennessy, E. J.; Ma, M.; Moore, K.; Farokhzad, O. C.; Fisher, E. A.; Mulder, W. J. M.; Langer, R.; Fayad, Z. A. Single Step Reconstitution of Multifunctional High-Density Lipoprotein-Derived Nanomaterials Using Microfluidics. ACS nano 2013, 7 (11), 9975-9983.

(44) Valencia, P. M.; Basto, P. A.; Zhang, L.; Rhee, M.; Langer, R.; Farokhzad, O. C.; Karnik, R. Single-step Assembly of Homogenous Lipid-polymeric and Lipid-quantum Dot Nanoparticles Enabled by Microfluidic Rapid Mixing. ACS nano 2010, 4 (3), 1671-1679. 
(45) Aucamp, M.; Odendaal, R.; Liebenberg, W.; Hamman, J. Amorphous Azithromycin with Improved Aqueous Solubility and Intestinal Membrane Permeability. Drug Dev. Ind. Pharm.2015, 41 (7), 1100-1108, DOI: $10.3109 / 03639045.2014 .931967$.

(46) Wan, F.; Nylander, T.; Klodzinska, S. N.; Foged, C.; Yang, M.; Baldursdottir, S. G.; H, M. N. Lipid ShellEnveloped Polymeric Nanoparticles with High Integrity of Lipid Shells Improve Mucus Penetration and Interaction with Cystic Fibrosis-Related Bacterial Biofilms. ACS Appl. Mater. Interfaces 2018, 10(13):1067810687, DOI: 10.1021/acsami.7b19762.

(47) Klausen, M.; Aaes-Jørgensen, A.; Molin, S.; Tolker-Nielsen, T. Involvement of Bacterial Migration in the Development of Complex Multicellular Structures in Pseudomonas aeruginosa Biofilms. Mol. Microbiol. 2003, 50 (1), 61-68, DOI: 10.1046/j.1365-2958.2003.03677.x.

(48) Wolter, J.; Seeney, S.; Bell, S.; Bowler, S.; Masel, P.; McCormack, J. Effect of Long Term Treatment with Azithromycin on Disease Parameters in Cystic Fibrosis: a Randomised Trial. Thorax. 2002, 57 (3), 212-216.

(49) Saiman, L.; Marshall, B.; Mayer-Hamblett, N.; Burns, J.; Quittner, A.; Cibene, D.; Coquillette, S.; Fieberg, A.; Accurso, F. Azithromycin in Patients with Cystic Fibrosis Chronically Infected with Pseudomonas aeruginosa: a Randomized Controlled Trial. JAMA 2003, 290 (13), 1749-1756.

(50) Jaffé, A.; Francis, J.; Rosenthal, M.; Bush, A. Long-term Azithromycin May Improve Lung Function in Children with Cystic Fibrosis. Lancet. 1998, 351 (9100), 420, DOI: 10.1016/s0140-6736(05)78360-4.

(51) Klodzinska, S. N.; Wan, F.; Jumaa, H.; Sternberg, C.; Rades, T.; Nielsen, H. M. Utilizing Nanoparticles for Improving Anti-biofilm Effects of Azithromycin: A Head-to-head Comparison of Modified Hyaluronic Acid Nanogels and Coated Poly (lactic-co-glycolic acid) Nanoparticles. J. Colloid Interface Sci. 2019, 555, 595-606, DOI: $10.1016 /$ j.jcis.2019.08.006.

(52) Pamp, S. J.; Gjermansen, M.; Johansen, H. K.; Tolker-Nielsen, T. Tolerance to the Antimicrobial Peptide Colistin in Pseudomonas aeruginosa Biofilms is Linked to Metabolically Active Cells, and Depends on the pmr and mexAB-oprM Genes. Mol. Microbiol. 2008, 68 (1), 223-240, DOI: 10.1111/j.1365-2958.2008.06152.x. (53) Niels Høiby. Understanding Bacterial Biofilms in Patients with Cystic Fibrosis: Current and Innovative Approaches to Potential Therapies. J. Cyst. Fibros. 2002, 1 (4), 249-254.

(54) Constantinou, C.; Papas, A.; Constantinou, A. I. Vitamin E and Cancer: An Insight into the Anticancer Activities of Vitamin E Isomers and Analogs. Int. J. Cancer 2008, 123 (4), 739-752, DOI: 10.1002/ijc.23689. (55) Danhier, F.; Ansorena, E.; Silva, J. M.; Coco, R.; Le Breton, A.; Preat, V. PLGA-based Nanoparticles: An Overview of Biomedical Applications. J. Control. Release 2012, 161 (2), 505-522, DOI:

10.1016/j.jconrel.2012.01.043.

(56) Fleet, J. E.; Guha, K.; Piper, S.; Banya, W.; Bilton, D.; Hodson, M. E. A Retrospective Analysis of the Impact of Azithromycin Maintenance Therapy on Adults Attending a UK Cystic Fibrosis Clinic. J. Cyst. Fibros. 2013, 12 (1), 49-53, DOI: 10.1016/j.jcf.2012.05.010.

(57) Levy, S. B.; Marshall, B. Antibacterial Resistance Worldwide: Causes, Challenges and Responses. Nat. Med. 2004, 10 (12 Suppl), S122-129, DOI: 10.1038/nm1145. 


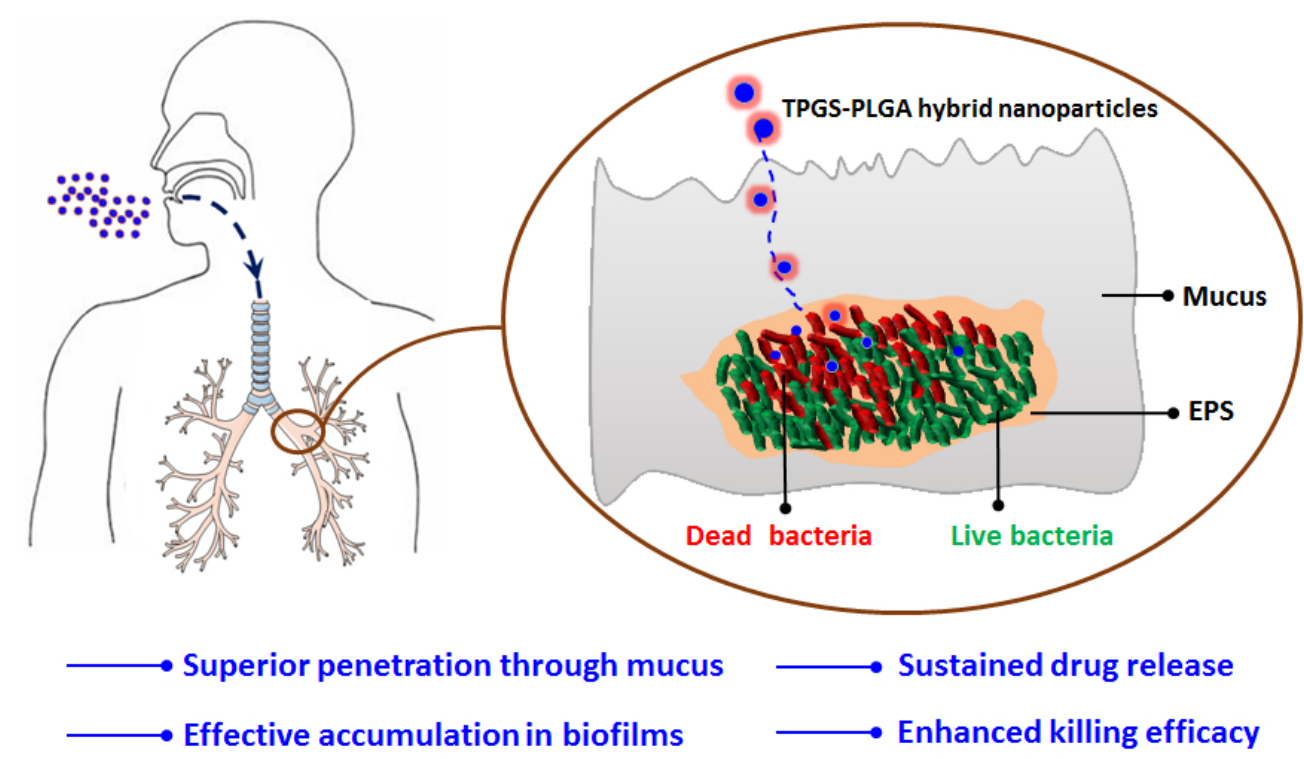

Graphic abstract

$231 \times 135 \mathrm{~mm}(96 \times 96 \mathrm{DPI})$ 